\title{
Application of Unsupervised Chemometric Analysis and Self-organising Feature Map (SOFM) for the Classification of Lighter Fuels
}

Wan N.S. Mat Desa, ${ }^{1}$ Niamh Nic Daéid, ${ }^{*}$ Dzulkiflee Ismail, ${ }^{1}$ and Kathleen Savage. Centre for Forensic Science, Department of Pure and Applied Chemistry, University of

Strathclyde, 204 George Street, Glasgow G1 1WX

* To whom correspondence should be addressed.

Phone: + 44-141-5484700. Fax: +44-141-5482532. E-mail: n.nicdaeid@strath.ac.uk.

${ }^{1}$ Permanent address: School of Health Sciences, Universiti Sains Malaysia, Health Campus, 16150 Kubang Kerian, Kelantan, Malaysia.

\begin{abstract}
A variety of lighter fuel samples from different manufacturers (both unevaporated and evaporated) were analysed using conventional gas chromatography-mass spectrometry (GCMS) analysis. In total 51 characteristic peaks were selected as variables and subjected to data pre-processing prior to subsequent analysis using unsupervised chemometric analysis (PCA and HCA) and a SOFM artificial neural network. The results obtained revealed that SOFM acted as a powerful means of evaluating and linking degraded ignitable liquid sample data to their parent unevaporated liquids.
\end{abstract}

